Review

\title{
Applications of Wireless Sensor Networks in Marine Environment Monitoring: A Survey
}

\author{
Guobao Xu ${ }^{1}$, Weiming Shen ${ }^{2, *}$ and Xianbin Wang ${ }^{2}$ \\ 1 Lab of Ocean Remote Sensing \& Information Technology, Guangdong Ocean University, \\ Zhanjiang 524088, China; E-Mail: xuguobao@126.com \\ 2 Department of Electrical and Computer Engineering, The University of Western Ontario, London, \\ Ontario N6A 5B9, Canada; E-Mail: xianbin.wang@uwo.ca \\ * Author to whom correspondence should be addressed; E-Mail: wshen@uwo.ca or wshen@iee.org; \\ Tel.: +1-519-430-7134; Fax: +1-519-430-7064.
}

Received: 9 July 2014; in revised form: 29 August 2014 / Accepted: 3 September 2014 /

Published: 11 September 2014

\begin{abstract}
With the rapid development of society and the economy, an increasing number of human activities have gradually destroyed the marine environment. Marine environment monitoring is a vital problem and has increasingly attracted a great deal of research and development attention. During the past decade, various marine environment monitoring systems have been developed. The traditional marine environment monitoring system using an oceanographic research vessel is expensive and time-consuming and has a low resolution both in time and space. Wireless Sensor Networks (WSNs) have recently been considered as potentially promising alternatives for monitoring marine environments since they have a number of advantages such as unmanned operation, easy deployment, real-time monitoring, and relatively low cost. This paper provides a comprehensive review of the state-of-the-art technologies in the field of marine environment monitoring using wireless sensor networks. It first describes application areas, a common architecture of WSN-based oceanographic monitoring systems, a general architecture of an oceanographic sensor node, sensing parameters and sensors, and wireless communication technologies. Then, it presents a detailed review of some related projects, systems, techniques, approaches and algorithms. It also discusses challenges and opportunities in the research, development, and deployment of wireless sensor networks for marine environment monitoring.
\end{abstract}


Keywords: wireless sensors; wireless sensor networks; marine environment monitoring; buoys; energy harvesting

\section{Introduction}

A wireless sensor network (WSN) consists of a number of dedicated sensor nodes with sensing and computing capabilities, which can sense and monitor the physical parameters and transmit the collected data to a central location using wireless communication technologies. A WSN has a number of inherent characteristics including uncontrollable environments, topological constraints, and limited node resources for energy and computational power [1]. Generally, a WSN deploys more sensors than the optimal placement in order to improve the system reliability and the fault tolerance [2].

During the last decade, WSNs have been widely utilized in a variety of application fields related to water monitoring [3-5], forest monitoring [6,7], industrial monitoring [8,9], agriculture monitoring [10,11], battlefield surveillance [12,13], intelligent transportation [14,15], smart homes [16,17], animal behavior monitoring [18,19], and disaster prevention [20,21]. This technology can certainly be applied to the monitoring of marine environments.

On the other hand, with the development of society and economy, more and more people have started to pay attention to the marine environment. Marine environment systems are particularly vulnerable to the effects of human activities related to industry, tourism and urban development [22]. Traditionally, oceanographic research vessels were used to monitor marine environments, which is a very expensive and time-consuming process that has a low resolution both in time and space. For marine environment research, a WSN-based approach can dramatically improve the access to real-time data covering long periods and large geographical areas [23]. According to Tateson et al. [24], a WSN-based approach is at least one order of magnitude cheaper than a conventional oceanographic research vessel.

In a WSN-based marine environment monitoring system, various kinds of sensors are used to monitor and measure different physical and chemical parameters such as water temperature, pressure, wind direction, wind speed, salinity, turbidity, $\mathrm{pH}$, oxygen density, and chlorophyll levels.

While the development and deployment of an adaptive, scalable and self-healing WSN system need to address a number of critical challenges such as autonomy, scalability, adaptability, self-healing and simplicity [25,26], the design and deployment of a lasting and scalable WSN for marine environment monitoring should take into account the following challenges different from those on land [22]:

(1) Higher water resistance: Sensor nodes of a marine monitoring system require greater levels of water resistance;

(2) Stronger robustness: A marine monitoring system needs stronger robustness, since the marine environment with waves, marine currents, tides, typhoons, vessels, etc., is aggressive and complex, and causes movement of nodes;

(3) Higher energy consumption: Energy consumption is higher due to long communication distances and an environment in constant motion; 
(4) More unstable line-of-sight: The oscillation of the radio antenna can cause a more unstable line-of-sight between transmitters and receivers [27].

(5) Other problems: There are also some other problems including the difficulty for deployment and maintenance of nodes, the need for buoy and mooring devices, sensor coverage problems [2], and possible acts of vandalism.

There have been a few literature reviews on Wireless Sensor Networks for marine environment monitoring. Albaladejo et al. [22] provided a comprehensive review of the research and development of oceanographic monitoring using wireless sensor networks and pointed out the challenges and difficulties of WSNs for oceanographic monitoring. This paper is intended as an update and extension of Albaladejo et al's review [22] based on recent developments in this area during the past five years. The limitations and challenges of wireless sensor networks for environmental research were discussed in [28]. They reviewed several WSN applications such as water ecosystems, forest monitoring, precision agriculture, wildlife observation, disaster prevention and urban monitoring.

This paper provides a comprehensive review of recent developments in the related fields, discusses major technical challenges, and identifies future research directions. The rest of the paper is organized as follows: Section 2 briefly describes fundamentals of WSN-based marine environment monitoring systems. Section 3 reviews some related projects, systems, and technologies. Section 4 highlights various challenges and opportunities including oceanographic sensors protection, advanced buoy design, energy harvesting system design, and WSN-based system stability and reliability. Section 5 provides some concluding remarks.

\section{Overview}

This section provides an overview on the application of WSNs in marine environment monitoring, including different application areas, a common architecture of WSN-based marine monitoring systems, a general architecture of an oceanographic sensor node, sensing parameters and sensors, and related wireless communication technologies.

\subsection{Application Areas}

WSN-based marine environment monitoring has a broad coverage including a number of application areas: water quality monitoring, ocean sensing and monitoring, coral reef monitoring, and marine fish farm monitoring. Different application areas require different WSN system architectures, communication technologies, and sensing technologies.

A water quality monitoring system is usually developed to monitor water conditions and qualities including temperature, $\mathrm{pH}$, turbidity, conductivity and dissolved oxygen (DO) for ocean bays, lakes, rivers and other water bodies. An ocean sensing and monitoring system is used to monitor ocean water conditions and other environmental parameters. A coral reef monitoring system is normally installed to monitor coral reef habitats using an autonomous, real-time and in-situ wireless sensor network. A marine fish farm monitoring system is developed to monitor water conditions and qualities including temperature and $\mathrm{pH}$, and accurately quantify the amount of fecal waste and uneaten feed for a fish farm. 


\subsection{Common WSN Architecture}

Figure 1 shows a common wireless sensor network architecture for monitoring marine environments, which consists of sensor nodes, sink nodes, a base station, a server and user terminals. Sensor nodes can sense and monitor the in-situ environmental parameters such as water temperature, salinity, turbidity, $\mathrm{pH}$, oxygen density and chlorophyll levels, and transmit the collected data to sink nodes via wireless communication using ZigBee or some other communication protocol. Communication between sensor nodes and a sink node is usually point-to-point. A sink node collects data from a group of sensor nodes, and transmits the collected data to the base station via the GPRS network. The server stores and processes the received data from the base station. The user terminals connect the server over the Internet.

Figure 1. Common architecture of WSN-based marine monitoring systems.

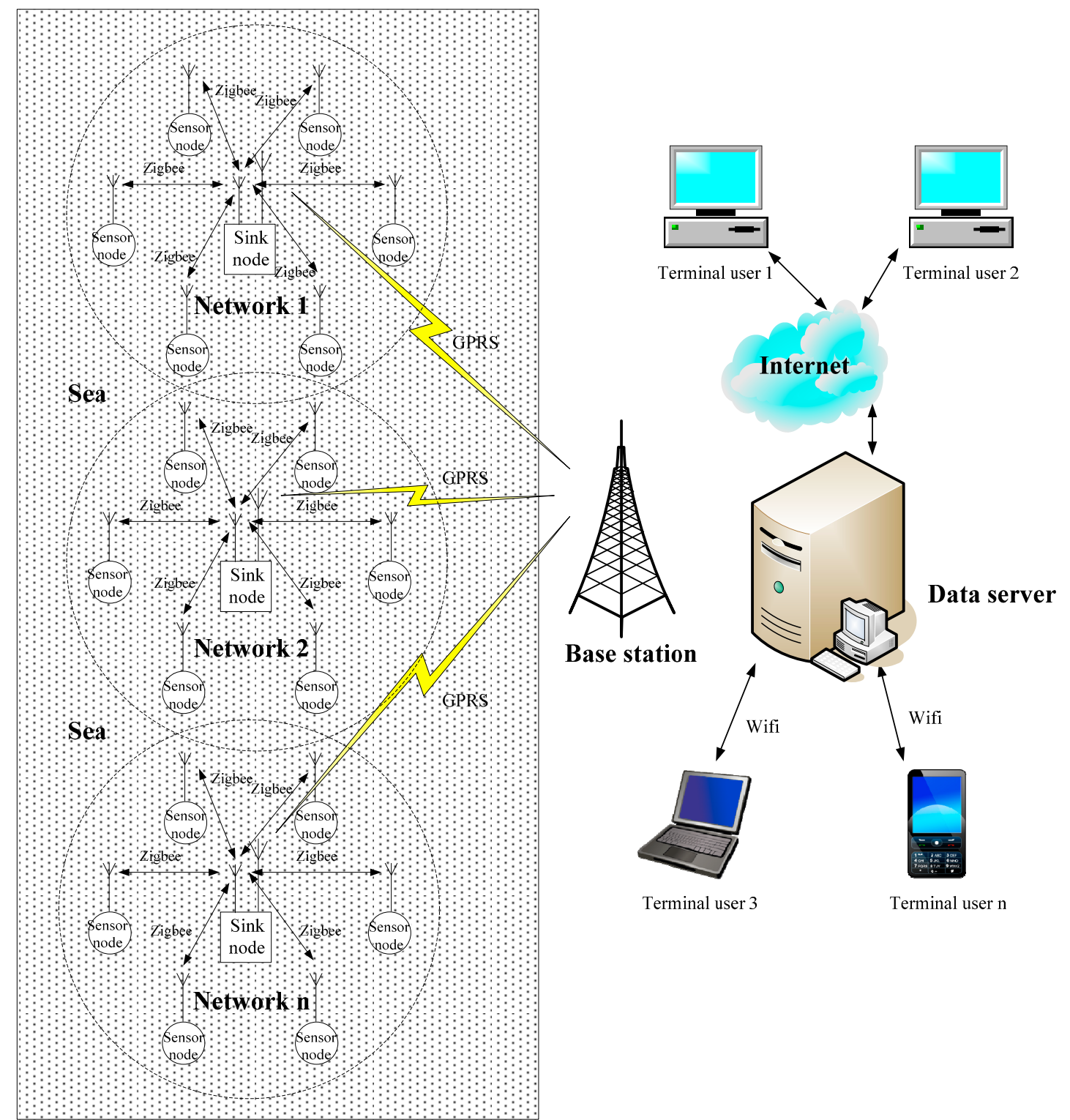

The design and deployment of a lasting and scalable WSN for marine environment monitoring should carefully take into account the following factors: the hostile environment, the network 
topology, communication protocols, the number of nodes, buoys, mooring systems, oceanographic sensors, energy supply, and so on.

\subsection{General Sensor Node}

Figure 2 shows an architecture of a general sensor node in a marine environment monitoring system. It usually includes a buoy device in order to protect electronic devices of nodes against water. A marine monitoring sensor node normally consists of the following four main modules [29]:

(1) A sensing module for data acquisition;

(2) A central processing module for local data processing and storage;

(3) A wireless transceiver module for wireless data communication;

(4) A power supply module for energy supply.

A sensing module is usually composed of several probes and sensors (with associated amplifiers and $\mathrm{A} / \mathrm{D}$ converters) to sense and monitor the physicochemical parameters of marine environment as mentioned above. A central processing module normally includes a CPU and memory to process and store the collected data. A wireless transceiver module mainly consists of a RF transceiver and an antenna to send the collected data and receive instructions from the sink node. A power supply module usually contains energy storage devices (rechargeable batteries), power management system and energy harvesting devices (solar panel, wind energy, tidal power, seawater generator, etc.). Finally the buoy has an anchor device in order to prevent it from moving (due to waves, marine currents, wind, tide, etc.).

Figure 2. General architecture of an oceanographic sensor node.

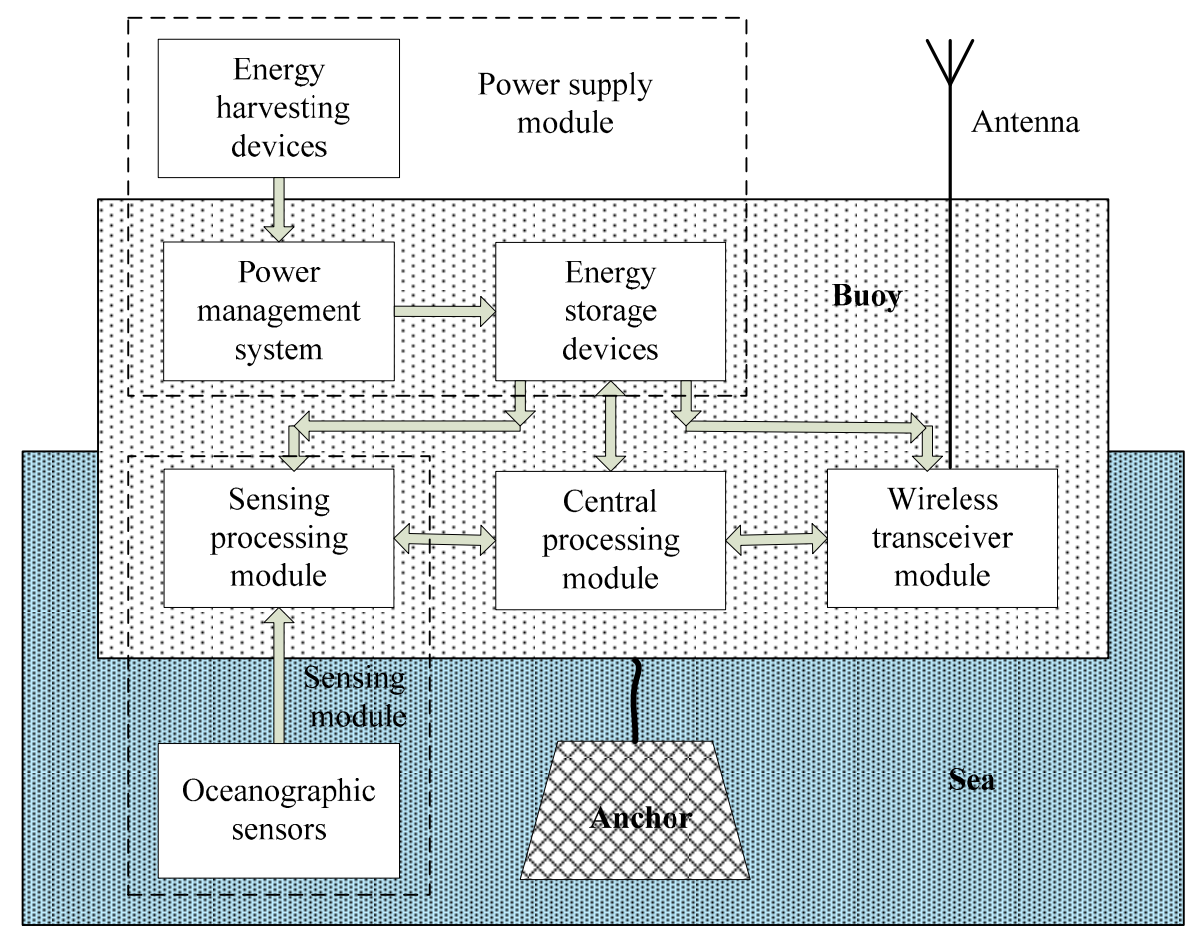


The energy options for sensor nodes usually include batteries, capacitors, heat engines, fuel cells, and energy harvesting. Sensor nodes are normally battery powered in most application systems. However, the use of a battery in sensor nodes has a number of disadvantages [30]:

(1) As sensor nodes increase in number and size, the replacement of depleted batteries is wasteful and time-consuming.

(2) A battery has limited energy that cannot last a long life for sensor nodes.

(3) Batteries have environmental contamination and disposal issues since the chemical composition of a battery often involves toxic heavy metals.

It is therefore necessary to explore an alternative power supply for sensor nodes. Harvesting energy from their ambient environment is a promising power supply for sensor networks with lower cost and long life. Energy harvesting methods include photovoltaics, fluid flow, temperature gradients, pressure variations and vibration harvesting. In terms of their efficiencies and realisability, the most outstanding energy harvesting at the moment is photovoltaics [30]. This issue will be further explored in Section 4.3.

\subsection{Sensing Parameters and Sensors}

The operating principle of sensors is to respond to changes in their environment by producing an electrical signal in the form of voltage, current, or frequency [31]. Sensors can commonly be divided into physical sensors and chemical sensors. In a marine monitoring system, physical sensors are used to measure some physical parameters, such as temperature, humidity, pressure, wind speed and wind direction, and chemical sensors are used to sense various chemical parameters (salinity, turbidity, $\mathrm{pH}$, nitrate, chlorophyll, dissolved oxygen (DO), etc.) as shown in Table 1.

Table 1. Common marine environment monitoring sensors.

\begin{tabular}{|c|c|c|c|c|c|c|}
\hline Sensors & $\begin{array}{l}\text { Monitoring } \\
\text { Parameters }\end{array}$ & Range & Accuracy & $\begin{array}{l}\text { Power } \\
\text { Supply }\end{array}$ & Unit & Manufacturer \\
\hline $\begin{array}{l}\text { SBE 16plus } \\
\text { V2 }\end{array}$ & Temperature & -5 to $+35^{\circ} \mathrm{C}$ & $\pm 0.005{ }^{\circ} \mathrm{C}$ & $9-28 \mathrm{~V}$ & ${ }^{\circ} \mathrm{C}$ & $\begin{array}{l}\text { Sea-Bird } \\
\text { Electronics }\end{array}$ \\
\hline GT301 & Pressure & 0 to 60 & $\begin{array}{l}< \pm 0.5 \% \text { of } \\
\text { FRO }\end{array}$ & $24 \mathrm{~V}$ & bar & $\begin{array}{l}\text { Kongsberg } \\
\text { Maritime }\end{array}$ \\
\hline $\begin{array}{l}\text { SBE 16plus } \\
\text { V2 }\end{array}$ & $\begin{array}{l}\text { Conductivity } \\
\text { (Salinity) }\end{array}$ & $0-9$ & \pm 0.0005 & $9-28 \mathrm{~V}$ & $\mathrm{~S} / \mathrm{m}$ & $\begin{array}{l}\text { Sea-Bird } \\
\text { Electronics }\end{array}$ \\
\hline OBS-3+ & Turbidity & $\begin{array}{l}\text { Mud: } 5000-10,000 \mathrm{mg} / \mathrm{L} \\
\text { Sand: } 50,000-100,000 \mathrm{mg} / \mathrm{L}\end{array}$ & $0.5 \mathrm{NTU}$ & $15 \mathrm{~V}$ & NTU & $\begin{array}{l}\text { Campbell } \\
\text { Scientic }\end{array}$ \\
\hline PS-2102 & $\mathrm{pH}$ & 0 to $14 \mathrm{pH}$ & \pm 0.1 & N/A & $\mathrm{pH}$ & PASCO \\
\hline YSI 6025 & Chlorophyll & 0 to $400 \mu \mathrm{g} / \mathrm{L}$ & $0.1 \mu \mathrm{g} / \mathrm{L}$ & $6 \mathrm{~V}$ & $\mu \mathrm{g} / \mathrm{L}$ & YSI \\
\hline ISUS V3 & Nitrate & 0.007 to $28 \mathrm{mg} / \mathrm{L}$ & $\pm 0.028 \mathrm{mg} / \mathrm{L}$ & $6-18 \mathrm{~V}$ & $\mathrm{mg} / \mathrm{L}$ & Satlantic \\
\hline SBE 63 & $\begin{array}{l}\text { Dissolved } \\
\text { oxygen (DO) }\end{array}$ & $\begin{array}{l}120 \% \text { of surface saturation } \\
\text { in all natural waters }\end{array}$ & 0.1 & $\begin{array}{l}6-24 \mathrm{~V} ; \\
35 \mathrm{~mA}\end{array}$ & $\mathrm{mg} / \mathrm{L}$ & $\begin{array}{l}\text { Sea-Bird } \\
\text { Electronics }\end{array}$ \\
\hline
\end{tabular}


The right choice of marine environment monitoring sensors depends on the user requirements of deployment area, measurement range, accuracy, resolution, power consumption, and intended deployment time.

\subsection{Wireless Communication Technologies}

WSN physical topology and density are entirely dependent on the applications [32], so the design and deployment of a WSN should consider its environment and application. A number of sensor nodes are densely deployed to improve data accuracy and achieve better system connectivity. However, a dense deployment of sensor nodes has some disadvantages: high energy consumption, data collisions, interferences, etc. [33]. WSN nodes normally have three typical kinds of network topologies: star topology, cluster/tree topology and mesh topology, as shown in Figure 3.

Figure 3. General WSN network topologies.
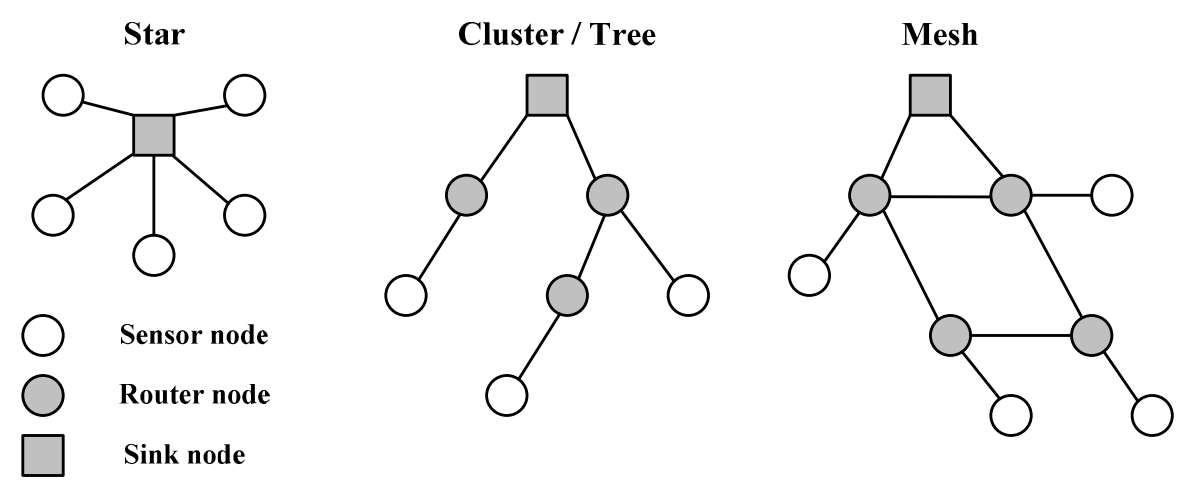

(1) Star topology: A star topology is a point-to-point single-hop architecture in which each sensor node connects directly to a sink node. It potentially uses the least amount of power among the three topology architectures.

(2) Mesh topology: A mesh topology is a one-to-many multi-hopping architecture in which each router node connects to multiple nodes. Its advantages over a star topology include a longer range distance of transmission, decreased loss of data, and a higher self-healing communication ability. However, its disadvantages are at the cost of higher latency and higher power consumptions.

(3) Cluster/tree topology: A cluster/tree topology is a hybrid star-mesh architecture. It takes advantage of the low power consumptions and simple architecture of a star topology, as well as the extended range and fault tolerance of a mesh one. However, there probably exists some latency.

The right and reasonable choice of network topology depends on the amount and frequency of data to be transmitted, transmission distance, battery life requirements and mobility of the sensor node [34]. It should be noted that a WSN physical topology may change due to available energy, position variations of nodes, malfunction, reachability (due to noise, severe weathers, moving obstacles, etc.), and task details of sensor nodes [35].

A sensor node normally incorporates a radio module for wireless communication. The transmitted distance of wireless communication can be anywhere between a few meters (Bluetooth, ZigBee, WiFi, etc.) and thousands of kilometers (GSM or GPRS radio communication). Wireless communication has various standards and technologies including Bluetooth, ZigBee, WiFi, GSM, GPRS and WiMAX. 
Table 2 provides a summary and brief comparison of these communication technologies. Usually, two or more wireless communication technologies are used in a real wireless sensor network. In particular, underwater acoustic communication technologies can be a good choice for data collection and exchange among underwater sensors [36-38].

Table 2. Wireless communication technologies [22].

\begin{tabular}{|c|c|c|c|c|c|}
\hline Technology & Standard & Description & Throughput & Range & Frequency \\
\hline $\mathrm{WiFi}$ & $\begin{array}{l}\text { IEEE } \\
802.11 \mathrm{a} ; \\
802.11 \mathrm{~b} / \mathrm{g} / \mathrm{n}\end{array}$ & $\begin{array}{l}\text { System of wireless data } \\
\text { transmission over computational } \\
\text { networks. }\end{array}$ & $\begin{array}{l}11 / 54 / 300 \\
\text { Mbps }\end{array}$ & $<100 \mathrm{~m}$ & $\begin{array}{l}5.8 \mathrm{GHz} \\
2.4 \mathrm{GHz}\end{array}$ \\
\hline Bluetooth & $\begin{array}{l}\text { IEEE } \\
802.15 .1\end{array}$ & $\begin{array}{l}\text { Industrial specification for WPAN } \\
\text { which enables voice and data } \\
\text { transmission between different } \\
\text { devices by means of a secure, } \\
\text { globally free radio link ( } 2.4 \mathrm{GHz}) \text {. }\end{array}$ & $\begin{array}{l}\text { v. } 1.2: 1 \mathrm{Mbps} \\
\text { v. } 2.0: 3 \mathrm{Mbps} \\
\text { UWB: } \\
\text { 53-480 Mbps }\end{array}$ & $\begin{array}{l}\text { Class 1: } 100 \mathrm{~m} \\
\text { Class 2: } 15-20 \\
\mathrm{~m} \\
\text { Class 3: } 1 \mathrm{~m}\end{array}$ & $2.4 \mathrm{GHz}$ \\
\hline ZigBee & $\begin{array}{l}\text { IEEE } \\
802.15 .4\end{array}$ & $\begin{array}{l}\text { Specification of a set of high-level } \\
\text { wireless communication protocols } \\
\text { for use with low consumption } \\
\text { digital radios, based on WPAN } \\
\text { standard IEEE 802.15.4. }\end{array}$ & $250 \mathrm{Kbps}$ & $<75 \mathrm{~m}$ & $2.4 \mathrm{GHz}$ \\
\hline WiMAX & $\begin{array}{l}\text { IEEE } \\
802.16\end{array}$ & $\begin{array}{l}\text { Standard for data transmission } \\
\text { using radio waves. }\end{array}$ & $<75 \mathrm{Mbps}$ & $<10 \mathrm{~km}$ & $2-11 \mathrm{GHz}$ \\
\hline GSM & & $\begin{array}{l}\text { Standard system for } \\
\text { communication via mobile } \\
\text { telephones incorporating digital } \\
\text { technology }\end{array}$ & $9.6 \mathrm{Kbps}$ & $\begin{array}{l}\text { Dependent on } \\
\text { service provider }\end{array}$ & $\begin{array}{l}850 / 900 / 1800 \\
/ 1900 \mathrm{MHz}\end{array}$ \\
\hline GPRS & & $\begin{array}{l}\text { GSM extension for unswitched (or } \\
\text { packaged) data transmission. }\end{array}$ & $56-144 \mathrm{Kbps}$ & $\begin{array}{l}\text { Dependent on } \\
\text { service provider }\end{array}$ & $\begin{array}{l}850 / 900 / 1800 \\
/ 1900 \mathrm{MHz}\end{array}$ \\
\hline
\end{tabular}

Generally, the longer the range a radio module must transmit, the more energy consumption a radio module will have. The choice of a wireless communication technology depends on the amount and frequency of the transmitted data, transmission distance, and amount of available energy.

\section{State-of-the-Art Review}

This section presents a comprehensive review of related projects, systems, applications, network routing mechanisms, algorithms, approaches and techniques on marine environment monitoring based on wireless sensor networks.

\subsection{Related Projects, Systems and Applications}

Different WSN projects, systems and applications have been proposed and developed in the literature for monitoring marine environments. Table 3 summarizes the features of related projects, systems and applications. 
Table 3. Summary of WSN-based marine environment monitoring projects, systems and applications.

\begin{tabular}{|c|c|c|c|c|c|c|c|c|c|c|}
\hline Reference & Organization & Country & Year & $\begin{array}{l}\text { Application } \\
\text { Areas }\end{array}$ & $\begin{array}{l}\text { Sensing } \\
\text { Parameters }\end{array}$ & $\begin{array}{l}\text { Communica- } \\
\text { tion Protocols }\end{array}$ & Buoy & $\begin{array}{l}\text { Energy } \\
\text { Harvesting }\end{array}$ & $\begin{array}{l}\text { Testing \& } \\
\text { Deployment }\end{array}$ & Main Features \\
\hline $\begin{array}{l}\text { Perez } \\
\text { et al. [4] }\end{array}$ & $\begin{array}{l}\text { Universidad } \\
\text { Politécnica de } \\
\text { Cartagena }\end{array}$ & Spain & 2011 & $\begin{array}{l}\text { Ocean sensing } \\
\& \text { monitoring }\end{array}$ & $\begin{array}{l}\text { Temperature, } \\
\text { pressure, salinity, } \\
\text { nitrates, velocity, } \\
\text { chlorophyll, and } \\
\text { turbidity }\end{array}$ & $\begin{array}{l}\text { GPRS } \\
\text { ZigBee }\end{array}$ & $\begin{array}{l}\text { Special } \\
\text { buoy }\end{array}$ & $\begin{array}{l}\text { Two solar } \\
\text { panels }\end{array}$ & $\begin{array}{l}\text { Deployed in } \\
\text { the harbor of } \\
\text { Cartagena }\end{array}$ & $\begin{array}{l}\text { LabVIEW-based user } \\
\text { interface using Google } \\
\text { Maps; Solar energy } \\
\text { harvesting; Special buoy }\end{array}$ \\
\hline $\begin{array}{l}\text { Thiemo } \\
\text { et al. [23] }\end{array}$ & $\begin{array}{l}\text { Swedish Institute } \\
\text { of Computer Sci. \& } \\
\text { University at } \\
\text { Berlin }\end{array}$ & $\begin{array}{l}\text { Sweden; } \\
\text { Germany }\end{array}$ & 2007 & $\begin{array}{l}\text { Ocean sensing } \\
\& \text { monitoring }\end{array}$ & $\begin{array}{l}\text { Temperature, } \\
\text { motion, vibration } \\
\text { and sound }\end{array}$ & GPRS & $\begin{array}{l}\text { Simple } \\
\text { buoy and } \\
\text { king's } \\
\text { buoy }\end{array}$ & Batteries & $\begin{array}{l}\text { Tested in } \\
\text { Baltic Sea }\end{array}$ & $\begin{array}{l}\text { Design of an advanced } \\
\text { low-cost buoy system }\end{array}$ \\
\hline $\begin{array}{l}\text { Yang } \\
\text { et al. [39] }\end{array}$ & $\begin{array}{l}\text { Penn State } \\
\text { University }\end{array}$ & USA & 2002 & $\begin{array}{l}\text { Water quality } \\
\text { monitoring }\end{array}$ & $\mathrm{pH}$ & $\begin{array}{l}\text { RF transceiver } \\
\text { and acoustic } \\
\text { transducer }\end{array}$ & $\begin{array}{l}\text { PVC } \\
\text { housing }\end{array}$ & $\begin{array}{l}\text { Two } \\
\text { rechargeabl } \\
\text { e batteries }\end{array}$ & $\begin{array}{l}\text { Lab testing } \\
\text { with } 5 \text { nodes }\end{array}$ & $\begin{array}{l}\text { The design of various } \\
\text { interface circuits and the } \\
\text { use of five air-based } \\
\text { sensor nodes }\end{array}$ \\
\hline $\begin{array}{l}\text { Vesecky } \\
\text { et al. }[40]\end{array}$ & $\begin{array}{l}\text { UC } \\
\text { Santa Cruz }\end{array}$ & USA & 2007 & $\begin{array}{l}\text { Ocean sensing } \\
\& \text { monitoring }\end{array}$ & $\begin{array}{l}\text { Temperature, } \\
\text { wave and } \\
\text { location }\end{array}$ & $900 \mathrm{MHz}$ & $\begin{array}{l}\text { mobile } \\
\text { minibuoy }\end{array}$ & $\begin{array}{l}\text { Battery } \\
\text { power }\end{array}$ & $\begin{array}{l}\text { Prototype } \\
\text { buoy tested } \\
\text { in a pool }\end{array}$ & $\begin{array}{l}\text { An autonomous mini- } \\
\text { buoy prototype; GPS and } \\
\text { a PID scheme control }\end{array}$ \\
\hline $\begin{array}{l}\text { Bromage } \\
\text { et al. }[41]\end{array}$ & UC Santa Cruz & USA & 2007 & $\begin{array}{l}\text { Coral reefs } \\
\text { monitoring }\end{array}$ & $\begin{array}{l}\text { Temperature, } \mathrm{pH} \text {, } \\
\text { light, pressure, } \\
\text { and conductivity }\end{array}$ & $900 \mathrm{MHz}$ & $\begin{array}{l}\text { Watertight } \\
\text { housing }\end{array}$ & Battery & $\begin{array}{l}\text { Monterey } \\
\text { Bay } \\
\text { deployment }\end{array}$ & $\begin{array}{l}\text { Programmable Oceanic } \\
\text { Device (POD) with a } \\
\text { 4-mode scheduler to save } \\
\text { energy }\end{array}$ \\
\hline $\begin{array}{l}\text { Seders } \\
\text { et al. [42] }\end{array}$ & $\begin{array}{l}\text { University of Notre } \\
\text { Dame }\end{array}$ & USA & 2007 & $\begin{array}{l}\text { Water quality } \\
\text { monitoring }\end{array}$ & $\begin{array}{l}\text { Temperature, } \mathrm{pH} \text {, } \\
\text { and DO }\end{array}$ & $433 \mathrm{MHz}$ & $\begin{array}{l}\text { Box and } \\
\text { polyethyle } \\
\text { ne ring }\end{array}$ & $\begin{array}{l}12 \text { volt } \\
\text { marine } \\
\text { battery }\end{array}$ & $\begin{array}{l}\text { Tested a } \\
\text { prototype in a } \\
\text { small lake }\end{array}$ & $\begin{array}{l}\text { A LakeNet sensor pod } \\
\text { and an altered sampling } \\
\text { strategy }\end{array}$ \\
\hline $\begin{array}{l}\text { Regan } \\
\text { et al. }[43]\end{array}$ & $\begin{array}{l}\text { Dublin City } \\
\text { University }\end{array}$ & Ireland & 2009 & $\begin{array}{l}\text { Water quality } \\
\text { monitoring }\end{array}$ & $\begin{array}{l}\text { Temperature, } \mathrm{pH} \text {, } \\
\text { turbidity, } \mathrm{DO} \text { and } \\
\text { conductivity }\end{array}$ & ZigBee & $\begin{array}{l}\text { Inshore } \\
\text { sensor } \\
\text { buoys }\end{array}$ & $\begin{array}{l}\text { Solar panel } \\
\text { and power } \\
\text { pack }\end{array}$ & $\begin{array}{l}\text { Deployed in } \\
\text { five sites on } \\
\text { the River } \\
\text { Lee, Ireland }\end{array}$ & $\begin{array}{l}\text { A real-time } \\
\text { heterogeneous water } \\
\text { quality monitoring; } \\
\text { Sensor maintenance }\end{array}$ \\
\hline
\end{tabular}


Table 3. Cont.

\begin{tabular}{|c|c|c|c|c|c|c|c|c|c|c|}
\hline $\begin{array}{l}\text { Liu et al. } \\
\text { [44] }\end{array}$ & $\begin{array}{l}\text { Hong Kong } \\
\text { University of Sci. } \\
\text { and Tech. }\end{array}$ & China & 2010 & $\begin{array}{l}\text { Ocean sensing } \\
\& \text { monitoring }\end{array}$ & $\begin{array}{l}\text { Sea depth and } \\
\text { temperature }\end{array}$ & ZigBee & $\begin{array}{l}\text { Sensor } \\
\text { floating }\end{array}$ & Batteries & $\begin{array}{l}\text { Deployed in } \\
\text { HKUST } \\
\text { campus and } \\
\text { Tsingtao }\end{array}$ & $\begin{array}{l}\text { A Perpendicular } \\
\text { Intersection (PI) mobile- } \\
\text { assisted localization } \\
\text { scheme }\end{array}$ \\
\hline $\begin{array}{l}\text { Lloret } \\
\text { et al. [45] }\end{array}$ & $\begin{array}{l}\text { Universidad } \\
\text { Politecnica de } \\
\text { Valencia, }\end{array}$ & Spain & 2011 & $\begin{array}{l}\text { Marine fish } \\
\text { farms } \\
\text { monitoring }\end{array}$ & $\begin{array}{l}\text { The amount of } \\
\text { pollution }\end{array}$ & $?$ & Buoy & $?$ & $\begin{array}{l}\text { Tested on } \\
\text { OPNET } \\
\text { Modeler } \\
\text { network } \\
\text { simulator }\end{array}$ & $\begin{array}{l}\text { A group-based } \\
\text { underwater WSN for } \\
\text { monitoring fecal waste } \\
\text { and uneaten feed }\end{array}$ \\
\hline $\begin{array}{l}\text { Macias } \\
\text { et al. }[46]\end{array}$ & $\begin{array}{l}\text { Universidad de Las } \\
\text { Palmas de Gran } \\
\text { Canaria }\end{array}$ & Spain & 2011 & $\begin{array}{l}\text { Ocean sensing } \\
\& \text { monitoring }\end{array}$ & $\begin{array}{l}\text { Visible-field, } \\
\text { sound and } \\
\text { temperature }\end{array}$ & $\begin{array}{l}\text { ZigBee and } \\
\text { acoustic }\end{array}$ & $?$ & $?$ & $\begin{array}{l}\text { Tested on } \\
\text { module of NS- } \\
3\end{array}$ & $\begin{array}{l}\text { Three tier } \\
\text { communication } \\
\text { architecture; transmitting } \\
\text { video streaming data }\end{array}$ \\
\hline $\begin{array}{l}\text { Roadknigh } \\
\text { t et al. } \\
{[47]}\end{array}$ & University of Kent & UK & 2004 & $\begin{array}{l}\text { Ocean sensing } \\
\& \text { monitoring }\end{array}$ & $\begin{array}{l}\text { Temperature, } \\
\text { conductivity, } \\
\text { water depth, } \\
\text { turbidity }\end{array}$ & $?$ & Single buoy & Batteries & $\begin{array}{l}\text { Buoy } \\
\text { deployed off } \\
\text { Scroby sands }\end{array}$ & $\begin{array}{l}\text { A multi-layered scalable } \\
\text { and adaptive approach of } \\
\text { data management }\end{array}$ \\
\hline $\begin{array}{l}\text { López } \\
\text { et al. [48] }\end{array}$ & $\begin{array}{l}\text { Universitat de } \\
\text { Barcelona }\end{array}$ & Spain & 2010 & $\begin{array}{l}\text { Fish farm } \\
\text { monitoring }\end{array}$ & $\begin{array}{l}\text { Temperature and } \\
\mathrm{pH}\end{array}$ & ZigBee & $?$ & $\begin{array}{l}\text { One } \\
\text { rechargeab } \\
\text { le battery }\end{array}$ & $\begin{array}{l}\text { Tested in a } \\
\text { pool }\end{array}$ & $\begin{array}{l}\text { A sub-layer-based power } \\
\text { consumption algorithm }\end{array}$ \\
\hline $\begin{array}{l}\text { O'Connor } \\
\text { et al. [49] }\end{array}$ & $\begin{array}{l}\text { Dublin City } \\
\text { University }\end{array}$ & Ireland & 2012 & $\begin{array}{l}\text { Water quality } \\
\text { monitoring }\end{array}$ & $\begin{array}{l}\text { Temperature, } \\
\text { conductivity and } \\
\text { depth }\end{array}$ & $?$ & Buoys & $?$ & $\begin{array}{l}\text { Tested in } \\
\text { River Lee, } \\
\text { Poolbeg } \\
\text { Marina and } \\
\text { Galway Bay }\end{array}$ & $\begin{array}{l}\text { A multi-modal } \\
\text { environment monitoring } \\
\text { network based on WSN } \\
\text { and visual image }\end{array}$ \\
\hline $\begin{array}{l}\text { Cella } \\
\text { et al. }[50]\end{array}$ & $\begin{array}{l}\text { University of } \\
\text { Queensland }\end{array}$ & Australia & 2009 & $\begin{array}{l}\text { Ocean sensing } \\
\& \text { monitoring }\end{array}$ & $\begin{array}{l}\text { Temperature and } \\
\text { illuminance }\end{array}$ & ZigBee & $\begin{array}{l}\text { Cylinder } \\
\text { waterproof } \\
\text { buoys }\end{array}$ & $\begin{array}{l}\text { Two solar } \\
\text { panels }\end{array}$ & $\begin{array}{l}\text { Deployed in } \\
\text { the Moreton } \\
\text { Bay }\end{array}$ & $\begin{array}{l}\text { Two solar cells and the } \\
\text { underwater wireless } \\
\text { communication }\end{array}$ \\
\hline $\begin{array}{l}\text { Diofantos } \\
\text { et al. }[51]\end{array}$ & $\begin{array}{l}\text { Cyprus University } \\
\text { of Technology }\end{array}$ & Cyprus & 2009 & $\begin{array}{l}\text { Water quality } \\
\text { monitoring }\end{array}$ & $\begin{array}{l}\text { Temperature, } \\
\text { pressure salinity } \\
\text { and turbidity }\end{array}$ & GPRS & $\begin{array}{l}\text { Cylinder } \\
\text { waterproof } \\
\text { buoy }\end{array}$ & Battery & $\begin{array}{l}\text { Deployed in a } \\
\text { municipal } \\
\text { beach }\end{array}$ & $\begin{array}{l}\text { Integrating two } \\
\text { technologies of satellite } \\
\text { remote sensing and WSN }\end{array}$ \\
\hline
\end{tabular}


Table 3. Cont

\begin{tabular}{|c|c|c|c|c|c|c|c|c|c|c|}
\hline $\begin{array}{l}\text { Yang } \\
\text { et al. [52] }\end{array}$ & $\begin{array}{l}\text { Zhejiang } \\
\text { University of } \\
\text { Technology }\end{array}$ & China & 2009 & $\begin{array}{l}\text { Monitoring } \\
\text { marine } \\
\text { shellfish }\end{array}$ & $\begin{array}{l}\text { Water } \\
\text { temperature, } \mathrm{pH} \\
\text { value, salinity, } \\
\text { DO and COD }\end{array}$ & GPRS & $?$ & $\begin{array}{l}\text { Solar } \\
\text { battery }\end{array}$ & $\begin{array}{l}\text { Tested in an } \\
\text { aquatic } \\
\text { experimental } \\
\text { base }\end{array}$ & $\begin{array}{l}\text { Multi-hop } \\
\text { communication protocol, } \\
\text { multiple nodes, and SMT }\end{array}$ \\
\hline $\begin{array}{l}\text { Jiang et al. } \\
\text { [53] }\end{array}$ & $\begin{array}{l}\text { Ocean University } \\
\text { of China }\end{array}$ & China & 2009 & $\begin{array}{l}\text { Ocean Sensing } \\
\& \text { monitoring }\end{array}$ & $\begin{array}{l}\text { Temperature, } \\
\text { velocity and light }\end{array}$ & ZigBee & Lever buoy & Battery & $\begin{array}{l}\text { Deployed off } \\
\text { the seashore }\end{array}$ & $\begin{array}{l}\text { The sleep mechanism } \\
\text { and lever buoy }\end{array}$ \\
\hline $\begin{array}{l}\text { Jin et al. } \\
{[54]}\end{array}$ & $\begin{array}{l}\text { China Jiliang } \\
\text { University }\end{array}$ & China & 2010 & $\begin{array}{l}\text { Water quality } \\
\text { monitoring }\end{array}$ & $\begin{array}{l}\text { Temperature, } \mathrm{pH}, \\
\mathrm{DO} \text {, and salinity }\end{array}$ & $\begin{array}{l}\text { ZigBee } \\
\text { GPRS }\end{array}$ & $?$ & Battery & $?$ & $\begin{array}{l}\text { Two wireless } \\
\text { communications of } \\
\text { ZigBee and GPRS }\end{array}$ \\
\hline $\begin{array}{l}\text { Chi et al. } \\
{[55]}\end{array}$ & $\begin{array}{l}\text { Shanghai Ocean } \\
\text { University }\end{array}$ & China & 2010 & $\begin{array}{l}\text { Ocean Sensing } \\
\& \text { monitoring }\end{array}$ & $\begin{array}{l}\text { Water } \\
\text { temperature, DO } \\
\text { and } \mathrm{pH}\end{array}$ & ZigBee & $\begin{array}{l}\text { Buoys with } \\
\text { GPS \&PEA }\end{array}$ & $?$ & $\begin{array}{l}\text { Experimented } \\
\text { in two testbeds }\end{array}$ & $\begin{array}{l}\text { Position determination } \\
\text { and location verification } \\
\text { using GPS\& PEA; Buoys }\end{array}$ \\
\hline $\begin{array}{l}\text { Cesare } \\
\text { et al. }[56]\end{array}$ & $\begin{array}{l}\text { Politecnico } \\
\text { di Milano, Milano }\end{array}$ & Italy & 2011 & $\begin{array}{l}\text { Ocean Sensing } \\
\& \text { monitoring }\end{array}$ & $\begin{array}{l}\text { Seawater } \\
\text { luminosity, } \\
\text { temperature and } \\
\text { moisture }\end{array}$ & ZigBee & $\begin{array}{l}\text { Cylinder } \\
\text { waterproof } \\
\text { buoys }\end{array}$ & $\begin{array}{l}\text { Solar } \\
\text { energy } \\
\text { harvesting }\end{array}$ & $\begin{array}{l}\text { Deployed in } \\
\text { the Moreton } \\
\text { Bay }\end{array}$ & $\begin{array}{l}\text { Optimal solar energy } \\
\text { harvesting; Power-aware } \\
\text { and adaptive TDMA } \\
\text { protocol }\end{array}$ \\
\hline $\begin{array}{l}\text { De } \\
\text { Marziani } \\
\text { et al. [57] }\end{array}$ & $\begin{array}{l}\text { National University } \\
\text { of Patagonia San } \\
\text { Juan Bosco }\end{array}$ & Argentina & 2011 & $\begin{array}{l}\text { Ocean Sensing } \\
\& \text { monitoring }\end{array}$ & $\begin{array}{l}\text { Temperature, } \\
\text { pressure, } \\
\text { PAR radiation, } \\
\text { pH and salinity }\end{array}$ & ZigBee & $\begin{array}{l}\text { Cylinder } \\
\text { waterproof } \\
\text { buoys }\end{array}$ & $\begin{array}{l}\text { Solar } \\
\text { panels }\end{array}$ & $\begin{array}{l}\text { Tested in San } \\
\text { Jorge Gulf }\end{array}$ & $\begin{array}{l}\text { A low cost } \\
\text { reconfigurable WSN; } \\
\text { Buoys; Solar panels }\end{array}$ \\
\hline $\begin{array}{l}\text { Alkandari } \\
\text { et al. }[58]\end{array}$ & Kuwait University & Kuwait & 2012 & $\begin{array}{l}\text { Water quality } \\
\text { monitoring }\end{array}$ & $\begin{array}{l}\text { Water } \\
\text { temperature, DO, } \\
\text { and } \mathrm{pH}\end{array}$ & $\begin{array}{l}\text { ZigBee } \\
802.11 \\
\text { Ethernet } \\
\text { radio }\end{array}$ & $?$ & $\begin{array}{l}\text { A high- } \\
\text { capacity } \\
\text { solar panel }\end{array}$ & $\begin{array}{l}\text { Tested in a } \\
\text { water pool }\end{array}$ & $\begin{array}{l}\text { Using ZigBee and } \\
802.11 \text { Ethernet radio } \\
\text { and a high capacity solar } \\
\text { panel }\end{array}$ \\
\hline $\begin{array}{l}\text { Albaladejo } \\
\text { et al. [59] }\end{array}$ & $\begin{array}{l}\text { Technical } \\
\text { University of } \\
\text { Cartagena }\end{array}$ & Spain & 2012 & $\begin{array}{l}\text { Ocean Sensing } \\
\& \text { monitoring }\end{array}$ & $\begin{array}{l}\text { Marine } \\
\text { temperature and } \\
\text { pressure }\end{array}$ & ZigBee & Special buoy & $\begin{array}{l}\text { Solar } \\
\text { panels }\end{array}$ & $\begin{array}{l}\text { Deployed in } \\
\text { Mar Menor } \\
\text { Lagoon }\end{array}$ & $\begin{array}{l}\text { A new multisensory } \\
\text { buoy system and solar } \\
\text { panels }\end{array}$ \\
\hline
\end{tabular}

Notes: ?: Related information is not available from the reference; DO: Dissolved Oxygen; COD: Chemical Oxygen Demand. 
It can be found that most of the efforts are related to general ocean sensing and monitoring [4,23,40,44,46,47,50,53,55-57] and water quality monitoring [39,42,43,49,51,54,58]. Some specific efforts have been made for fish farm monitoring [45,48], coral reef monitoring [41], and marine shellfish monitoring [52]. Some projects focus specific technologies or devices, e.g., buoys [23,40,53,57,59] which will be further discussed in Section 4.2, and energy saving and harvesting [4,43,48,50,52,56-59], discussed in details in Section 4.3.

Most developed systems were only experimented in lab settings or indoor environments [39,45,46,52,55], some are tested in outdoor pools or small ponds/lakes [40,42,48,58], and a number of them have been tested or deployed in real marine or river environments $[4,23,41,43,44,47,49-51,53,57,59]$.

It is also interesting to note that during the last decade most projects, systems and applications have been developed by research groups in a small number of countries, including USA [39,40,41,42], China [44,52-55], Spain [4,45,46,48,59] and Ireland [43,49].

\subsection{Specific Networks, Routing Mechanisms and Algorithms}

To satisfy the requirements for marine environment monitoring systems, researchers have proposed and developed a number of specific networks, routing mechanisms, protocols, and algorithms for WSN-based marine environment monitoring.

A WSN-based data collection framework was proposed and developed by Saha et al. [60] for disaster mitigation and rescue operation. A WSN communication protocol with lower delay and better energy efficiency was proposed for data dissemination from disaster areas. A simulation experiment was conducted to validate the performance of the proposed protocol comparing with the SENDROM system protocol.

A group-based underwater wireless sensor network (UWSN) was proposed by Lloret et al. [45] to monitor accurately the amount of fecal waste and uneaten feed deposited on the seabed which can cause the damage of the fauna and flora. The design and development of this underwater WSN took into account several factors: number of sensor nodes, sensor nodes mobility model, distribution of sensor nodes, network topology, and communication technologies.

Roadknight et al. [47] proposed a multi-layered scalable and adaptive approach of data management for a wireless sensor network. This algorithm consisted of three decision making components: sliding window averaging, local rules and parameter evolution. A single buoy was deployed off Scroby Sands to verify the characteristics of the proposed approach.

A WSN framework was proposed by Lu et al. [61] for environmental monitoring applications. Its highlight is on its network layer design by considering multiple aspects: heterogeneity, service-aware control platform, unified routing and scheduling, network monitoring. A special case study was conducted to demonstrate that the framework can be used to guide how to design a WSN for environment monitoring in the future.

Barbosa et al. [62] presented a routing algorithm of WSNs for marine oil slick monitoring. They proposed two methods: single relay decision (SRD) and multiple relay decision (MRD) protocols for message routing. The proposed algorithms have more efficient message distribution than single hop and greedy approaches. However, their approach does not consider node mobility, energy harvesting and network scalability. 
An IEEE 802.15.4-based wireless monitoring system was presented by López et al. [48] to collect $\mathrm{pH}$ and temperature parameters in a fish farm. The proposed algorithm used a ZigBee-based routing and the application layer to manage information transmission from the source node to the central coordinator. They designed a sub-layer-based power consumption algorithm to prolong the node lifetime.

$\mathrm{Xu}$ et al. [63] proposed an improved WSN MAC protocol for marine environment monitoring to meet the demand of the energy consumption, real-time transmission, bandwidth and reliability. Simulation results show good energy consumption and network throughput abilities. However, the proposed algorithm was not implemented in the actual sensor node to verify its performance.

A WSN dedicated dynamic clustering algorithm was presented for oil slicks monitoring by Harchi et al. [64]. It can be applied to a monitoring system adaptively in terms of number of nodes, clustering dynamics, measurement periods, and metric weights to supervise climate conditions. Various parameters are evaluated regarding their influence on the stability of the network clustering algorithm.

Suakanto et al. [65] proposed a cloud computing-based approach for data processing in disaster monitoring. The proposed approach used a FTR-HTTP based delivery method from remote client to server.

Jalali et al. [66] proposed a cooperative hybrid ARQ (C-HARQ) mechanism in solar powered wireless sensor networks to improve energy efficiency and reliability of energy harvesting. They conducted C-HARQ experiments using a Matlab/Simulink-based simulator for networked and embedded systems. Their experimental results showed that C-HARQ is superior to C-ARQ in energy consumption of relay nodes.

\subsection{Specific Techniques and Approaches}

To address the special needs and purposes of marine environment monitoring, a number of WSN-based techniques and approaches have been developed and reported in the literature.

O'Connor et al. [67] presented a multi-modal event monitoring system based on WSNs and visual images for river and coastal marine detection. The system used a visual sensor to complement the use of a WSN in detecting and tracking features of a river or coastal marine location. A software tool was developed to analyze the relationship between the sensor readings and image features. It uses a support vector machine (SVM) approach for training or classification. A Matlab image processing toolbox was used for processing images and extracting various image features including color features, texture features, and edge features.

Kong et al. [68] designed a WSN-based water environment monitoring system which can sense and monitor video information in key areas and various water quality parameters, including water temperature, turbidity, $\mathrm{pH}$, dissolved oxygen and electric conductivity. This monitoring system has a data video base station, data monitoring nodes, and a remote monitoring center. This system used an ARM-DSP based double processor, combined ZigBee and CDMA wireless transmission networks, and used a CPLD sampling controller.

A decentralized ad-hoc wireless sensor network was proposed for ocean pollution detection by Khan et al. [69]. In order to prolong the network lifetime and to improve its Quality of Service (QoS), they focused on the deployment of sensors, protocol stacks, synchronization and the routing algorithms. 
A WSN-based wave monitoring technology was proposed by Marin-Perianu et al. [70] to monitor various wave parameters. This system deployed dense wireless sensor nodes which are equipped with low-cost, low-power, MEMS-based inertial sensors of accelerometers and gyroscopes. They conducted experiments using a Ferris wheel contraption and the results showed an accuracy of approximately $10 \mathrm{~cm}$ for a wheel diameter of $100 \mathrm{~cm}$.

A robotic wireless sensor network was presented by Bhadauria et al. [71] for monitoring common carp in Minnesota lakes. This project built a small, mobile, lightweight robotic raft which is deployed with searching and tracking algorithms. They conducted several field experiments in various lakes, and experimental results demonstrated that the robotic raft has great potential in environmental monitoring. They envisioned some system improvements including energy saving, localization accuracy, autonomous navigation and multi-raft systems.

In order to enhance measurement precision and prolong the lifetime of marine environmental monitoring sensors, Delauney et al. [72] analyzed the biofouling effects on marine sensors measurements, proposed some promising techniques for the biofouling protection of in situ sensors.

To explore the impact of the deep ocean increase in $\mathrm{CO}_{2}$ levels and resulting $\mathrm{pH}$ changes on ocean biogeochemistry and ecology, Herlien et al. [73] studied a Free Ocean $\mathrm{CO}_{2}$ Enrichment (FOCE) system. The proposed algorithm can achieve the objectives of instrument-in-the-loop control, software reuse of infrastructure and instrument services, and rapid assembly of a scalable end-to-end sensor network system.

An Android-based WSN application was developed by Tembekar [74]. This WSN app can be installed and operate on any Android-based smartphone, get synchronized with the centralized database server, and monitor the various nodes of the wireless sensor network.

\section{Research Challenges and Opportunities}

So far, wireless sensor networks have been widely applied to terrestrial areas, and some of these deployments have achieved satisfactory performance. However, the application of WSNs in marine environment monitoring is still in its infancy, and most WSN-based systems are purely experimental [22]. This section discusses a few challenges of wireless sensor networks for marine environment monitoring including oceanographic sensors protection, advanced buoy design, energy harvesting system design, and system stability and reliability.

\subsection{Oceanographic Sensors Protection}

In marine environments, there are over 4000 organisms related to fouling problems [75]. According to their sizes, organisms can be classified into micro-organisms (or so called biofilms, slimes, and micro-fouling) and macro-fouling [72]. Biofouling development on a sensor surface is subject to several chemical, physical and biological factors such as $\mathrm{pH}$, dissolved oxygen, temperature, light, location depth, conductivity, organic material and hydrodynamic conditions. When oceanographic sensors are immersed in seawater, they are susceptible to biofouling problems which often lead to the long-term accuracy issues of marine environmental sensor measurements. Since the marine environment is aggressive and the seawater is corrosive, oceanographic sensors should take appropriate fouling protection measures. 
The biofouling protection for oceanographic sensors may be divided into three techniques according to their different actions: wipers mechanisms, copper corrosion mechanisms, and chlorine evolution mechanisms [72].

(1) Wiper mechanisms: A biofouling protection system based on wipers is a purely mechanical method. It is an effective biofouling protection technique as long as the sensor head has a suitable shape for wiper cleaning and the wipers are in good condition.

(2) Copper corrosion mechanisms: A copper corrosion mechanism is an effective biofouling protection method to protect the sensitive sensor head, but the protection mechanism is not easy to apply to existing sensors and the cost is relatively high.

(3) Chlorine evolution mechanisms: A biofouling protection system based on a chlorine evolution mechanism uses bleach or chlorine generation by seawater electrolysis. Moreover, this protection mechanism is easily adapted to existing sensors and the cost is relatively low.

Besides the abovementioned three biofouling protection techniques, there are some other interesting methods which promise effective results coming from research laboratories [76,77]. However, it is very difficult to implement these methods in the real sea environment.

Biofouling protection for oceanographic in-situ sensors is a very difficult problem. The ideal biofouling protection for oceanographic sensors should take into account six aspects: low cost, low power consumption, easy to install on existing sensors, no or low impact on measurement precision and the environment, long lifetime and robustness against aggressive conditions. Therefore, researchers and manufacturers should further study and explore the biofouling protection mechanisms for marine environmental sensors.

\subsection{Advanced Buoy Design}

Considering the marine environment is aggressive and complex, it is very crucial to design an advanced flotation device (buoy) for a marine environment monitoring system. A buoy normally consists of a wireless sensor network node (CPU, sensors, radio, and batteries), an energy harvesting module, underwater sensors and a mooring system. For example, Pirisi et al. [78] proposed a special energy harvesting buoy which can effectively use sea wave energy conversion as a power source and has potential applications in marine environment monitoring. Albaladejo et al. [59] designed a multisensory buoy system which can be effectively used for shallow marine environment monitoring.

The design and deployment of an advanced buoy for marine wireless sensor networks should take into account the following requirements: low cost, waterproof, strong stability, energy harvesting, and mooring system.

(1) Low cost: A marine environment monitoring system using wireless sensor networks is usually composed of a large number of sensor nodes. Therefore, each buoy device needs to be low cost.

(2) Watertightness: In order to protect the stability of marine environment monitoring system and prolong its lifetime, its electronic devices must be in a waterproof housing to avoid water damage.

(3) Strong stability: As the marine environment is aggressive and complex, the monitoring system should have a strong stability against adverse atmospheric conditions. 
(4) Energy harvesting: Since it is not convenient to replace the batteries deployed on the marine surface and the sensor nodes, which are far away from the land and are power-hungry, it is necessary to consider the use of energy harvesting to reduce system maintenance requirements.

(5) Mooring system: Due to tides, waves, marine currents, wind, etc., an anchor is required on the seabed in order to avoid the movement of the buoy devices.

Besides the above mentioned requirements, the buoy mechanic design should meet a number of requirements including the buoy visibility with bright yellow color and a warning light for maritime traffic, the use of environmentally friend materials, the connection of several sensors, and the reasonable antenna height for the better communication propagation.

\subsection{Energy Harvesting System Design}

The energy supply of a wireless sensor network is generally provided by batteries which have limited energy [27]. In addition, in marine environment monitoring systems, wireless sensor nodes are often deployed in unapproachable sea surface areas, and they are mostly planned for long-time operation, therefore, it is not convenient to replace the sensor batteries. Moreover, marine sensor nodes (sink nodes) have high energy consumption due to the use of long-range wireless communication protocol (GPRS). In order to reduce system maintenance requirements effectively, there is a clear need to design an energy harvesting system which uses renewable energies source such as solar [4], tidal power [78], or wind energy [79,80].

Some energy harvesting devices have been designed and developed to prolong the lifetime of marine environment monitoring systems. For example, Perez et al. [4] developed a solar energy harvesting device which is composed of two solar panels behind the electronic equipment with an inclination of 45 degrees in an opposite direction. In order that one battery is always being charged while the other is always being discharged, a power management system of a low-power maximum power point tracker (MPPT) circuit was developed and used for wireless sensor networks in [56] and [81].

To design an advanced energy harvesting system for marine environment monitoring, we should consider the following three aspects: energy harvesting devices, power management system, and energy storage devices.

- Energy harvesting devices: An energy harvesting device is responsible for harvesting energy from the ambient environment. According to the characteristics of available ambient energies, we should choose appropriate energy harvesting devices and should consider how to install the energy harvesting devices.

- Power management system: A power management system can intelligently manage the batteries to be charged and discharged at separate intervals of time. An ideal power management system can prolong the lifetime of batteries and easily store more energy for the system.

- Energy storage devices: Energy storage devices normally adopt the rechargeable batteries. Usually, the energy capacity of rechargeable batteries is larger than daily system energy consumption and daily harvesting energy in order to store energy and permit the system to supply power even in case of bad weather [56]. 
Given the aggressive and hostile marine environment, in order to harvest and use more reliable renewable energies, we can envision a hybrid harvesting energy system for marine environment monitoring in the future, which can use several renewable power sources such as solar, tidal power, seawater generator, and wind energy.

\subsection{System Stability and Reliability}

During the past decade, the system stability and reliability problem of wireless sensor networks has been widely studied in order to measure physical parameters correctly and effectively, as well as to prolong the lifetime of the system dramatically [82,83]. AboElFotoh et al. [84] studied the reliability and message delay for cooperative wireless distributed sensor networks subject to random failures. Egeland et al. [85] analyzed the reliability and availability of wireless multi-hop networks with stochastic link failures. Qureshi et al. [86] presented a methodology using a topology control mechanism for the reliability evaluation of a WSN. Silva et al. [8] proposed a methodology based on an automatic generation of a fault tree to evaluate the reliability and availability of wireless sensor networks in typical industrial environments.

Considering the aggressive and complex environment, it is very important to analyze the system reliability in a marine environmental monitoring system using wireless sensor networks. Therefore, the research on the reliability of a WSN-based marine environment monitoring system should take into account the following aspects.

(1) Battery life issues: As mentioned above, marine sensor nodes (sink nodes) consume more energy than other kinds of wireless sensor nodes. Therefore, the battery life issue always affects the system reliability.

(2) Communication relay issue: The communication relay affects dramatically the system reliability, when some nodes fail or simply disappear.

(3) Severe environment conditions: The marine environment always has external interference from ships, fishes, and birds, and has severe weather conditions such as waves, marine currents, tides and typhoons. Such severe environment conditions further influence the system reliability.

\section{Conclusions}

During the last decade, monitoring of the marine environment has attracted a great deal of research and development attention. Wireless sensor networks are a highly promising technique for monitoring marine environments because of their advantages of easy deployment, real-time monitoring, automatic operation, and low cost. This paper presents a state-of-the-art survey of applications of wireless sensor networks in marine environment monitoring. It first describes fundamentals of WSNs-based marine environment monitoring, including application areas, a common WSN architecture, a general sensor node architecture, sensing parameters and sensors, and wireless communication technologies. Then, it reviews the related literature according to different projects, systems, applications, network routing mechanisms, algorithms, approaches and techniques on marine environment monitoring based on wireless sensor networks. From this survey, it is evident that there are still a few interesting challenges and opportunities on development and deployment of wireless sensor networks for marine 
environment monitoring, including oceanographic sensors protection, advanced buoy design, energy harvesting system design, and system stability and reliability.

\section{Acknowledgments}

This work was financially supported by the Guangdong Ocean University scholarship council, and the Guangdong Ocean University Projects (E09174) to establish Ph.D. graduate programs.

\section{Conflicts of Interest}

The authors declare no conflict of interest.

\section{References}

1. Li, N.; Zhang, N.; Das, S.K.; Thuraisingham, B. Privacy preservation in wireless sensor networks: A state-of-the-art survey. Ad Hoc Netw. 2009, 7, 1501-1514.

2. Cardei, M.; Wu, J. Energy-efficient coverage problems in wireless ad-hoc sensor networks. Comput. Commun. 2006, 29, 413-420.

3. Lee, H.C.; Banerjee, A.; Fang, Y.M.; Lee, B.J.; King, C.T. Design of a multifunctional wireless sensor for in situ monitoring of debris flows. IEEE Trans. Instrum. Meas. 2010, 59, 2958-2967.

4. Perez, C.A.; Jimenez, M.; Soto, F.; Torres, R.; López, J.A.; Iborra, A. A system for monitoring marine environments based on Wireless Sensor Networks. In Proceedings of the IEEE Conference on OCEANS, Santander, Spain, 6-9 June 2011; pp. 1-6.

5. Jiang, P.; Xia, H.; He, Z.; Wang, Z. Design of a Water Environment Monitoring System Based on Wireless Sensor Networks. Sensors 2009, 9, 6411-6434.

6. Bayo, A.; Antolín, D.; Medrano, N.; Calvo, B.; Celma, S. Early Detection and Monitoring of Forest Fire with a Wireless Sensor Network System. Proced. Eng. 2010, 5, 248-251.

7. Yunus, E.A.; Ibrahim, K.; Özgür, U. A framework for use of wireless sensor networks in forest fire detection and monitoring. Comput. Environ. Urban Syst. 2012, 36, 614-625.

8. Silva, I.; Guedes, L.A.; Portugal, P.; Vasques, F. Reliability and Availability Evaluation of Wireless Sensor Networks for Industrial Applications. Sensors 2012, 12, 806-838.

9. Zhao, G. Wireless Sensor Networks for Industrial Process Monitoring and Control: A Survey. Netw. Protoc. Algorithms 2011, 3, 46-63.

10. Raul, M.; Samuel, G.M.; Miguel, A.F.; António, L.G.V.; Salviano, F.S.P.S.; Ferreira, P.J.S.G.; Reis, M.J.C.S. Sun, wind and water flow as energy supply for small stationary data acquisition platforms. Comput. Electron. Agric. 2008, 64, 120-132.

11. Li, X.; Deng, Y.; Ding, L. Study on precision agriculture monitoring framework based on wsn. In Proceedings of the 2nd International Conference on Anti-counterfeiting, Security and Identification (ASID 2008), Guiyang, China, 20-23 August 2008; pp. 182-185.

12. Qian, H.; Sun, P.; Rong, Y. Design Proposal of Self-Powered WSN Node for Battle Field Surveillance. Energy Proced. 2012, 16, 753-757.

13. Padmavathi, G.; Shanmugapriya, D.; Kalaivani, M. A Study on Vehicle Detection and Tracking Using Wireless Sensor Networks. Wirel. Sens. Netw. 2010, 2, 173-185. 
14. Tacconi, D.; Miorandi, D.; Carreras, I.; Chiti, F.; Fantacci, R. Using wireless sensor networks to support intelligent transportation systems. Ad Hoc Netw. 2010, 8, 462-473.

15. Tubaishat, M.; Zhuang, P.; Qi, Q.; Shang, Y. Wireless sensor networks in intelligent transportation systems. Wirel. Commun. Mob. Comput. 2009, 9, 287-302.

16. Lee, H.; Wu, C.; Aghajan, H. Vision-based user-centric light control for smart environments. Pervasive Mob. Comput. 2011, 7, 223-240.

17. Bangali, J.; Shaligram, A. Energy efficient Smart home based on Wireless Sensor Network using LabVIEW. Am. J. Eng. Res. 2013, 2, 409-413.

18. Handcock, R.N.; Swain, D.L.; Bishop-Hurley, G.J.; Patison, K.P.; Wark, T.; Valencia, P. Monitoring Animal Behaviour and Environmental Interactions Using Wireless Sensor Networks, GPS Collars and Satellite Remote Sensing. Sensors 2009, 9, 3586-3603.

19. Nadimi, E.S.; Jørgensen, R.N., Blanes-Vidal, V.; Christensen, S. Monitoring and classifying animal behavior using ZigBee-based mobile ad hoc wireless sensor networks and artificial neural networks. Comput. Electron. Agric. 2012, 82, 44-54.

20. Bahrepour, M.; Meratnia, N.; Poel, M.; Taghikhaki, Z.; Havinga, P.J.M. Distributed event detection in wireless sensor networks for disaster management. In Proceedings of the 2010 2nd International Conference on Intelligent Networking and Collaborative Systems (INCOS), Thessaloniki, Greece, 24-26 November 2010; pp. 507-512.

21. Lacono, M.; Romano, E.; Marrone, S. Adaptive monitoring of marine disasters with intelligent mobile sensor networks. In Proceedings of the 2010 IEEE Workshop on Environmental Energy and Structural Monitoring Systems (EESMS), Taranto, Italy, 9 September 2010; pp. 38-45.

22. Albaladejo, C.; Sánchez, P.; Iborra, A.; Soto, F.; López, J.A.; Torres, R. Wireless Sensor Networks for Oceanographic Monitoring: A Systematic Review. Sensors 2010, 10, 6948-6968.

23. Thiemo, V.; Fredrik, O.S.; Niclas, F. Sensor Networking in Aquatic Environments-Experiences and New Challenges. In Proceedings of the 32nd IEEE Conference on Local Computer Networks, Dublin, Ireland, 15-18 October 2007; pp. 793-798.

24. Tateson, J.; Roadknight, C.; Gonzalez, A.; Khan, T.; Fitz, S.; Henning, I. Real World Issues in Deploying a Wireless Sensor Network for Oceanography. In Proceedings of the Workshop on Real-World Wireless Sensor Networks, Stockholm, Sweden, 20-21 June 2005; pp. 20-21.

25. Boonma, P.; Suzuki, J. An Adaptive, Scalable and Self-Healing Sensor Network Architecture for Autonomous Coastal Environmental Monitoring. In Proceedings of the IEEE Conference on Technologies For Homeland Security, Woburn, MA, USA, 16-17 May 2007; pp. 1-8.

26. Hadim, S.; Mohamed, N. Middleware challenges and approaches for wireless sensor networks. IEEE Distrib. Syst. Online 2006, 7, 853-865.

27. Alippi, C.; Camplani, R.; Galperti, C.; Roveri, M. Effective design of WSNs: from the lab to the real world. In Proceedings of the 3rd International Conference on Sensing Technology, Tainan, Taiwan, 30 November-3 December 2008; pp. 1-9.

28. De la Piedra, A.; Benitez-Capistros, F.; Dominguez, F.; Touhafi, A. Wireless sensor networks for environmental research: A survey on limitations and challenges. In Proceedings of the 2013 IEEE Conference on EUROCON, Zagreb, Yugoslavia, 1-4 July 2013; pp. 267-274.

29. Giuseppe, A.; Marco, C.; Mario, D.F.; Andrea, P. Energy conservation in wireless sensor networks: A survey. Ad Hoc Netw. 2009, 7, 537-568. 
30. Knight, C.; Davidson, J.; Behrens, S. Energy Options for Wireless Sensor Nodes. Sensors 2008, 8, 8037-8066.

31. Porter, J.; Arzberger, P.; Braun, H.W.; Bryant, P.; Gage, S.; Hansen, T.; Hanson, P.; Lin, C.C.; Lin, F.P.; Kratz, T.; Michener, W.; Shapiro, S.;Williams, T. Wireless Sensor Networks for Ecology. BioScience 2005, 55, 561-572.

32. Li, M.; Yang, B. A Survey on topology issues in wireless sensor networks. In Proceedings of the 4th International Symposium on Information Processing in Sensor Networks, Las Vegas, NV, USA, 25-27 April 2005; pp. 1-7.

33. Zeng, Y.; Sreenan, C.J.; Xiong, N.; Yang, L.T.; Park, J.H. Connectivity and coverage maintenance in wireless sensor networks. J. Supercomput. 2010, 52, 23-46.

34. Flammini, A.; Ferrari, P.; Marioli, D.; Sisinni, E.; Taroni, A. Wired and wireless sensor networks for industrial applications. Microelectron. J. 2009, 40, 1322-1336.

35. Kim, S.; Guzide, O.; Cook, S. Towards an Optimal Network Topology in Wireless Sensor Networks: A Hybrid Approach. In Proceedings of the ISCA First International Conference on Sensor Networks and Applications, San Francisco, CA, USA, 4-6 November 2009; pp. 13-18.

36. Jiang, Z. User Density in a Cluster Underwater Acoustic Network. Int. J. Intell. Control Syst. 2012, 17, 31-40.

37. Jiang, Z. Underwater Acoustic Networks - Issues and Solutions. Int. J. Intell. Control Syst. 2008, $13,152-161$.

38. Zhu, Y.; Jiang, Z.; Peng, Z.; Zuba, M.; Cui, J.; Chen, H. Towards Practical MAC Design for Underwater Acoustic Networks. In Proceedings of the 32nd IEEE International Conference of Computer Communications (INFOCOM) 2013, Turin, Italy, 14-19 April 2013; pp. 683-691.

39. Yang, X.; Ong, K.G.; Dreschel, W.R.; Zeng, K.; Mungle, C.S.; Grimes, C.A. Design of a wireless sensor network for long-term, in-situ monitoring of an aqueous environment. Sensors 2002, 2, 455-472.

40. Vesecky, J.F.; Laws, K.; Petersen, S.I.; Bazeghi, C.; Wiberg, D. Prototype autonomous mini-buoy for use in a wireless networked, ocean surface sensor array. In Proceedings of the IEEE International Geoscience and Remote Sensing Symposium, Barcelona, Spain, 23-28 July 2007; pp. 4987-4990.

41. Bromage, M.; Obraczka, K.; Potts, D. SEA-LABS: A wireless sensor network for sustained monitoring of coral reefs. In Proceedings of the 6th international IFIP-TC6 conference on Ad Hoc and sensor networks, wireless networks, next generation internet, Atlanta, GA, USA, 14-18 May 2007; pp. 1132-1135.

42. Seders, L.A.; Shea, C.A.; Lemmon, M.D.; Maurice, P.A.; Talley, J.W. LakeNet: An Integrated Sensor Network for Environmental Sensing in Lakes. Environ. Eng. Sci. 2007, 24, 183-191.

43. Regan, F.; Lawlor, A.; Flynn, B.O.; Torres, J.; Martinez-Catala, R.; O’Mathuna, C.; Wallace, J. A demonstration of wireless sensing for long term monitoring of water quality. In Proceedings of the IEEE 34th Conference on Local Computer Networks, Zurich, Switzerland, 20-23 October 2009; pp. 819-825.

44. Liu, K.; Yang, Z.; Li, M.; Guo, Z.; Guo, Y.; Hong, F.; Yang, X.; He, Y.; Feng, Y.; Liu, Y. Oceansense: Monitoring the sea with wireless sensor networks. Mob. Comput. Commun. Rev. 2010, 14, 7-9. 
45. Lloret, J.; Sendra, S.; Garcia, M.; Lloret, G. Group-based Underwater Wireless Sensor Network for Marine Fish Farms. In Proceedings of the 2011 IEEE GLOBECOM Workshops, Houston, TX, USA, 5-9 December 2011; pp. 115-119.

46. Macias, E.; Suarez, A; Chiti, F.; Sacco, A.; Fantacci, R. A Hierarchical Communication Architecture for Oceanic Surveillance Applications. Sensors 2011, 11, 11343-11356.

47. Roadknight, C.; Parrott, L.; Boyd, N.; Marshall, I.W. A Layered Approach to in situ Data Management on a Wireless Sensor Network. In Proceedings of the International Conference on Intelligent Sensors, Sensor Networks and Information Processing, Melbourne, Australia, 14-17 December 2004; pp. 85-90.

48. López, M.; Gómez, J.M.; Sabater, J.; Herms, A. IEEE 802.15.4 based wireless monitoring of pH and temperature in a fish farm. In Proceedings of the 15th IEEE Mediterranean Electrotechnical Conference on MELECON 2010, Valletta, Malta, 26-28 April 2010; pp. 575-580.

49. O’Connor, E.; Zhang, D.; Smeaton, A.F.; O’Connor, N.E.; Regan, F. Multi-Modal Sensor Networks for More Effective Sensing in Irish Coastal and Freshwater Environments. In Proceedings of the IEEE Oceans, Hampton Roads, VA, USA, 14-19 October 2012; pp. 1-9.

50. Cella, U.M.; Shuley, N.; Johnstone, R. Wireless Sensor Networks in Coastal Marine Environments: a Study Case Outcome. In Proceedings of the Fourth ACM International Workshop on UnderWater Networks, Berkeley, CA, USA, 4-6 November 2009; pp. 1-8.

51. Diofantos, G.H.; Marinos, G.H.; Kyriacos, T.; Athos, A. Integration of micro-sensor technology and remote sensing for monitoring coastal water quality in a municipal beach and other areas in Cyprus. In Proceedings of the SPIE Remote Sensing for Agriculture, Ecosystems, and Hydrology, Berlin, Germany, 18 September 2009.

52. Yang, H.; Wu, H.; He, Y. Architecture of Wireless Sensor Network for Monitoring Aquatic Environment of Marine Shellfish. In Proceedings of the 7th Asian Control Conference, Hong Kong, China, 27-29 August 2009; pp. 1147-1151.

53. Jiang, M.; Guo, Z.; Hong, F.; Ma, Y.; Luo, H. OceanSense: A Practical Wireless Sensor Network on the Surface of the Sea. In Proceedings of the IEEE International Conference on Pervasive Computing and Communications, Galveston, TX, USA, 9-13 March 2009; pp. 1-5.

54. Jin, N.; Ma, R.; Lv, Y.; Lou, X.; Wei, Q. A Novel Design of Water Environment Monitoring System Based on WSN. In Proceedings of the 2010 International Conference on Computer Design and Applications (ICCDA), Qinhuangdao, China, 25-27 June 2010; pp. 593-597.

55. Chi, T.; Zhang, H.; Chen, M.; Feng, G. Implementation Study of a Home Position Monitoring System for Marine Environment. In Proceedings of the 2nd IEEE International Conference on Information Management and Engineering (ICIME), Chengdu, China, 16-18 April 2010; pp. 231-224.

56. Cesare, A.; Romolo, C.; Cristian, G.; Manuel, R. A Robust, Adaptive, Solar-Powered WSN Framework for Aquatic Environmental Monitoring. IEEE Sens. J. 2011, 11, 45-55.

57. De Marziani, C.; Alcoleas, R.; Colombo, F.; Costa, N.; Pujana, F.; Colombo, A.; Aparicio, J.; Álvarez, F.J.; Jimenez, A.; Ureña, J.; Hernández, A. A low cost reconfigurable sensor network for coastal monitoring. In Proceedings of the IEEE Conference on OCEANS, Santander, Spain, 6-9 June 2011; pp. 1-6. 
58. Alkandari, A.; Alnasheet, M.; Alabduljader, Y.; Moein, S.M. Water monitoring system using Wireless Sensor Network (WSN): Case study of Kuwait beaches. In Proceedings of the Second International Conference on Digital Information Processing and Communications (ICDIPC), Klaipeda City, Lithuania, 10-12 July 2012; pp. 10-15.

59. Albaladejo, C.; Soto, F.; Torres, R.; Sánchez, P.; López, J.A. A low-cost sensor buoy system for monitoring shallow marine environments. Sensors 2012, 12, 9613-9634.

60. Saha, S.; Matsumoto, M. A Framework for Disaster Management System and WSN Protocol for Rescue Operation. In Proceedings of the IEEE Region 10 Conference on TENCON 2007, Taipei, Taiwan, 30 October-2 November 2007; pp. 1-4.

61. Lu, K.; Qian, Y.; Rodriguez, D.; Rivera, W.; Rodriguez, M. Wireless Sensor Networks for Environmental Monitoring Applications: A Design Framework. In Proceedings of the Global Communications Conference, Washington, DC, USA, 26-30 November 2007; pp. 1108-1112.

62. Barbosa, P.; White, N.M.; Harris, N.R. Wireless Sensor Network for Localized Maritime Monitoring. In Proceedings of the 22nd International Conference on Advanced Information Networking and Applications, Okinawa, Japan, 25-28 March 2008; pp. 681-686.

63. Xu, L.; Zhang, J.; Li, H.H.; Ye, P.; Yan, X.; Li, X. An Improved MAC Protocal for Marine-Environment Monitoring WSN System. J. Netw. 2012, 7, 1900-1907.

64. Harchi, S.; Georges, J.; Divoux, T. WSN dynamic clustering for oil slicks monitoring. In Proceedings of the 2012 International Conference on Wireless Communications in Unusual and Confined Areas (ICWCUCA), Clermont Ferrand, France, 28-30 August 2012; pp. 1-6.

65. Suakanto, S.; Supangkat, S.H.; Suhardi; Saragih, R.; Nugroho, T.A.; Nugraha, I.G.B.B. Environmental and Disaster Sensing Using Cloud Computing Infrastructure. In Proceedings of the 2012 International Conference on Cloud Computing and Social Networking, Bandung, West Java, 26-27 April 2012; pp. 1-6.

66. Jalali, F.; Khodadoustan, S.; Ejlali, A. Cooperative Hybrid ARQ in Solar Powered Wireless Sensor Networks. Microelectron. Reliab. 2012, 52, 3043-3052.

67. O’Connor, E.; Smeaton, A.F.; O’Connor, N.E. A multi-modal event detection system for river and coastal marine monitoring applications. In Proceedings of the 2011 IEEE OCEANS, Santander, Spain, 6-9 June 2011; pp. 1-10.

68. Kong, Y.; Jiang, P. Development of Data Video Base Station in Water Environment Monitoring Oriented Wireless Sensor Networks. In Proceedings of the International Conference on Embedded Software and Systems Symposia, Sichuan, China, 29-31 July 2008; pp. 281-286.

69. Khan, A.; Jenkins, L. Undersea wireless sensor network for ocean pollution prevention. In Proceedings of the 3rd International Conference on Communication Systems Software and Middleware and Workshops, COMSWARE 2008, Bangalore, India, 6-10 January 2008; pp. 2-8.

70. Marin-Perianu, M.; Chatterjea, S.; Marin-Perianu, R.; Bosch, S.; Dulman, S.; Kininmonth, S. Wave Monitoring with Wireless Sensor Networks. In Proceedings of the International Conference on Intelligent Sensors, Sensor Networks and Information Processing, Sydney, Australia, 15-18 December 2008; pp. 611-616.

71. Bhadauria, D.; Isler, V.; Studenski, A.; Tokekar, P. A Robotic Sensor Network for Monitoring Carp in Minnesota Lakes. In Proceedings of the 2010 IEEE International Conference on Robotics and Automation, Anchorage, AK, USA, 3-7 May 2010; pp. 3837-3842. 
72. Delauney, L.; Compère, C.; Lehaitre, M. Biofouling protection for marine environmental sensors. Ocean Sci. 2010, 6, 503-511.

73. Herlien, R.; O’Reilly, T.; Headley, K.; Edgington, D.R.; Tilak, S.; Fountain, T.; Shin, P. An ocean observatory sensor network application. In Proceedings of the 2010 IEEE Conference on Sensors, Kona, HI, USA, 1-4 November 2010; pp. 1837-1842.

74. Tembekar, S.; Saxena, A. Monitoring Wireless Sensor Network using Android based SmartPhone Application. IOSR J. Comput. Eng. 2014, 16, 53-57.

75. Yebra, D.M.; Kiil, S.; Dam-Johansen, K. Antifouling technology—past, present and future steps towards efficient and environmentally friendly antifouling coatings. Prog. Org. Coat. 2004, 50, 75-104.

76. Whelan, A.; Regan, F. Antifouling strategies for marine and riverine sensors. J. Environ. Monit. 2006, 8, 880-886.

77. Manov, D.V.; Chang, G.C.; Dickey, T.D. Methods for reducing biofouling of moored optical sensors. J. Atmos. Ocean. Technol. 2004, 21, 958-968.

78. Pirisi, A.; Grimaccia, F.; Mussetta, M.; Zich, R.E.; Johnstone, R.; Palaniswami, M. Optimization of an Energy Harvesting Buoy for Coral Reef Monitoring. In Proceedings of the 2013 IEEE Congress on Evolutionary Computation, Cancún, Mexico, 20-23 June 2013; pp. 629-634.

79. Hsu, C.L. Constructing transmitting interface of running parameters of small-scaled wind-power electricity generator with WSN modules. Expert Syst. Appl. 2010, 37, 3893-3909.

80. Seah, W.K.G.; Zhi, A.E.; Tan, H. Wireless sensor networks powered by ambient energy harvesting (WSN-HEAP) - Survey and challenges. In Proceedings of the 1st International Conference on Wireless Communication, Vehicular Technology, Information Theory and Aerospace \& Electronic Systems Technology, Aalborg, Denmark, 17-20 May 2009; pp. 1-5.

81. Cesare, A.; Cristian, G. An adaptive system for optimal solar energy harvesting in wireless sensor network nodes. IEEE Trans. Circuits Syst. I Regul. Pap. 2008, 55, 1742-1750.

82. Islam, K.; Shen, W.; Wang, X. Wireless Sensor Network Reliability and Security in Factory Automation: A Survey. IEEE Trans. Syst. Man Cybern. Part C 2012, 42, 1243-1256.

83. Shen, Z.; Man, K.L.; Lei, C.U.; Lim, E.G.; Choi, J. Assuring System Reliability in Wireless Sensor Networks Via Verification and Validation. In Proceedings of the 2012 International on SoC Design Conference (ISOCC), Jeju Island, Korea, 4-7 November 2012; pp. 285-288.

84. AboElFotoh, H.M.F.; Iyengar, S.S.; Chakrabarty, K. Computing reliability and message delay for cooperative wireless distributed sensor networks subject to random failures. IEEE Trans. Reliab. 2005, 54, 145-155.

85. Egeland, G.; Engelstad, P. The availability and reliability of wireless multi-hop networks with stochastic link failures. IEEE J. Sel. Areas Commun. 2009, 27, 1132-1146.

86. Qureshi, H.K.; Rizvi, S.; Saleem, M.; Khayam, S.A.; Rakocevic, V.; Rajarajan, M. Poly: A reliable and energy efficient topology control protocol for wireless sensor networks. Comput. Commun. 2011, 34, 1235-1242.

(C) 2014 by the authors; licensee MDPI, Basel, Switzerland. This article is an open access article distributed under the terms and conditions of the Creative Commons Attribution license (http://creativecommons.org/licenses/by/3.0/). 